\title{
Composition Dependence of the Viscoelasticity of End-Linked Poly(dimethylsiloxane) at the Gel Point
}

\author{
James C. Scanlan and H. Henning Winter* \\ Department of Chemical Engineering, University of Massachusetts, \\ Amherst, Massachusetts 01003
}

Received February 7, 1990; Revised Manuscript Received May 21, 1990

\begin{abstract}
Precursor composition is shown to have a considerable impact on the viscoelastic properties of a polymer at the gel point. End-linked poly(dimethylsiloxane) polymers were prepared with various stoichiometry, chain length, and concentration. The power law relaxation modulus, $G(t)=S t^{-n}$, identifies the gel point, and a small frequency window ( 2 decades) is sufficient to determine the relaxation exponent, $n$, and gel strength, $S$. Both are shown to be strongly composition dependent. The relaxation exponent ranges between 0.19 and 0.92 in this study, which is almost the entire possible range $(0<n<1)$. The gel strength ranges over 5 decades. A prepolymer below the entanglement molecular weight produces critical gels with relaxation exponents in the range $0.4-0.7$, depending on stoichiometry. A high molecular weight prepolymer, above the entanglement molecular weight, produces critical gels with lower $n$ values, in the range of 0.2-0.4, depending on stoichiometry. Addition of a diluent also affects the relaxation exponent, and near the overlap concentration $n$ approaches the limit of 1 . In a series of experiments with different stoichiometry and concentration, $S$ and $n$ appear to be related by a material characteristic function $S=G_{0} \lambda_{0}^{n}$, where $G_{0}$ and $\lambda_{0}$ are characteristic constants of the precursor material and $n$ depends on composition.
\end{abstract}

\section{Introduction}

Recent dynamic mechanical experiments on crosslinking polymers lead to a new mechanical definition of the gel point (GP) ${ }^{1-3}$ The material at GP, termed the critical gel (CG), is at an intermediate state between liquid and solid. The CG was shown to have a power law relaxation modulus

$$
G(t)=S t^{-n}, \text { at GP }
$$

where the gel strength, $S$, and relaxation exponent, $n$, are the only material parameters. The relaxation exponent is physically restricted for $0<n<1$. The gel strength has rather unusual dimensions $\left(\mathrm{Pa} \mathrm{s}{ }^{n}\right)$, which will be investigated experimentally in this study.

Power law behavior is also apparent in dynamic mechanical experiments. The storage modulus, $G^{\prime}$, and the loss modulus, $G^{\prime \prime}$, at $G P$ are

$G^{\prime}(\omega)=G^{\prime \prime}(\omega) / \tan (n \pi / 2)=\Gamma(1-n) \cos (n \pi / 2) S \omega^{n}$

The phase angle between stress and strain, $\delta$, is independent of frequency but proportional to the relaxation exponent

$$
\delta=n \pi / 2
$$

Power law mechanical behavior is an expression of mechanical self-similarity and is a consequence of structural self-similarity, which evolves during cross-linking. The range of power law has not been determined, although we expect it to predominate on length scales from as small as the prepolymer strand length up to the macroscopic sample dimension.

The determination of the relaxation exponent remains an important test of our understanding of polymer dynamics. The dynamical properties should be intimately related to a material's structure. Theories of gelation describe the cluster growth process. ${ }^{4}$ They predict a power law cluster number distribution at the gel point in terms of the cluster mass, $M$

$$
N_{M} \sim M^{-\tau}, \text { at GP }
$$

where $\tau$ is termed the polydispersity index. The cluster radius is also expected to be a power law at the gel point

$$
R_{M} \sim M^{1 / d_{f}}, \text { at GP }
$$

with exponent $d_{f}$, which is the fractal dimension. The critical gel is thought to be statistically self-similar over many decades of mass.

Muthukumar ${ }^{5}$ and Cates ${ }^{6}$ derived an expression for the relaxation exponent in terms of the fractal dimension for a monodisperse solution of polymers when Rouse dynamics prevail and found

$$
n=d_{\mathrm{f}} /\left(d_{\mathrm{f}}+2\right)
$$

If the fractal dimension is not universal but arbitrary for $1 \leq d_{\mathrm{f}} \leq 3$, then this theory predicts that the relaxation exponent is restricted for $1 / 3 \leq n \leq 3 / 5$. This model has been applied to experimental results. ${ }^{7}$

Corrections for the polydispersity of the critical gel have been made. ${ }^{8,9}$ In this case the relaxation exponent becomes

$$
n=d_{\mathrm{f}}(\tau-1) /\left(d_{\mathrm{f}}+2\right)
$$

The prediction using the percolation statistics is

$$
n=2 / 3
$$

If, however, the fractal dimension is arbitrary, then the relaxation exponent is restricted for $3 / 5 \leq n \leq 1$ for $1 \leq$ $d_{\mathrm{f}} \leq 3$.

Hess et al..$^{10}$ and Muthukumar ${ }^{11}$ suggested that cluster growth models using rigid bonds may not properly describe the dimensions of the CG. Under the influence of excluded volume, Hess et al. suggested that flexible clusters may swell from the percolation prediction to $d_{f}=2$. The prediction using eq 6 becomes

$$
n=1 / 2
$$

The degree of the excluded-volume effect may depend on the chain length and other compositional variables. If excluded volume is completely screened, then the prediction for a polydisperse material becomes

$$
n=\frac{d\left(d+2-2 d_{\mathrm{f}}\right)}{2\left(d+2-d_{\mathrm{f}}\right)}
$$

Partial screening of excluded volume would result in values 
Table I

Literature Values of the Relaxation Exponent with Materials and Composition Used*

\begin{tabular}{lllll}
\hline \multicolumn{1}{c}{ system } & $r$ & $X_{\mathrm{n}}$ & \multicolumn{1}{c}{$n$} & ref \\
\hline PDMS & 0.7 & $\sim 135$ & 0.58 & $1-3$ \\
& 1.0 & & 0.5 & \\
polyurethane & $\geq 1$ & $\sim 17$ & 0.5 & 13,34 \\
& $\leq 1$ & & $0.5-0.7$ & \\
polyurethane & 0.55 & $\sim 10-20$ & 0.7 & 8 \\
epoxy & & & 0.7 & 9 \\
polyester & & 4 & 0.7 & 14 \\
radiation-cured & & & 0.2 & 16 \\
$\quad$ PE & & & 0.8 & 15 \\
$10 \%$ PVC & & & \\
$\quad$ physical gel & & & 0.18 & 17
\end{tabular}

a Compositional variables include the stoichiometry of the reaction, $r$, and the initial degree of polymerization of the prepolymer, $X_{n}$.

for $n$ between those in eqs 7 and 10. All values of the relaxation exponent for $0<n<1$ are possible for a fractal dimension in the physically acceptable range $1 \leq d_{\mathfrak{f}} \leq 3$.

Recent studies of critical gel rheological properties are summarized in Table I. Data have been reported in support of both predictions, $n=1 / 2$ and $n=2 / 3$. Chambon and Winter ${ }^{1-3}$ studied a poly(dimethylsiloxane) (PDMS) of degree of polymerization $X_{\mathrm{n}} \sim 135$ and found the relaxation exponent to depend on stoichiometry. Stoichiometric ratios of $r=0.7$ and 1 yielded values of $n$ of 0.58 and 0.5 , respectively. Stoichiometry strongly affects the final modulus of the network rubber. ${ }^{12}$ When crosslinking $f$-functional $A_{f}$ molecules with $g$-functional $B_{g}$ molecules, the molar stoichiometry

$$
r=f\left[A_{\mathrm{f}}\right] / g\left[B_{g}\right]
$$

has to be chosen between a lower and upper critical value

$$
r_{1}=[(f-1)(g-1)]^{-1}, \quad r_{\mathrm{u}}=1 / r_{1}
$$

in order for the material to reach the gel point. A polyurethane (PU) system ${ }^{13}$ with $X_{\mathrm{n}} \sim 17$ yielded $n$ values between $1 / 2$ and $2 / 3$, also depending on stoichiometry. On the other hand, several groups have found only a value close to $2 / 3$. Durand et al. ${ }^{8}$ report a value of $n=0.69 \pm$ 0.04 , also for a low molecular weight polyurethane, but only reported the deficient stoichiometry, $r_{1}$, that just yields a critical gel. Martin et al. ${ }^{9}$ found a value of $n=$ $0.70 \pm 0.05$ for an epoxy. Rubinstein et al. ${ }^{14}$ report $n=$ $0.69 \pm 0.02$ for bulk condensation of a polyester. Effects of composition or structure were not observed by any of these groups. Values outside the range $1 / 2^{-2} / 3$ have also been found. Nijenhuis and Winter ${ }^{15}$ found a value of $n$ $\approx 0.8$ for a physically cross-linked PVC plastisol system. Valles ${ }^{16}$ reports $n \approx 0.2$ for a vulcanized PE, and Lin et al. ${ }^{17}$ report of value of $n \approx 0.2$ for a crystallizing polypropylene.

Due to the critical nature of the gel point, we initially expected a universal value of the relaxation exponent. However, in light of the experimental evidence, we believe now that a universal value of the relaxation exponent does not exist. Therefore, we have set out to elucidate the nature of the relationship between the composition and structure of a CG to its dynamic behavior. We present here studies of the effects of stoichiometry, chain length, and dilution on the CG linear viscoelastic properties.

\section{Experimental Section}

Materials and Characterization. Various divinyl-terminated poly (dimethylsiloxane) prepolymers were end-linked by a hydrosilation reaction ${ }^{18}$ with a four-functional cross-linker, tetrakis (dimethylsiloxy)silane. The materials were obtained from
Table II

Properties of the Materials Used in This Study*

\begin{tabular}{cccccc}
\hline & & \multicolumn{2}{c}{$X_{\mathrm{n}}$} & & \\
\cline { 3 - 4 } material & $f$ assumed & GPC & titration & $X_{\mathrm{w}} / X_{\mathrm{n}}$ (GPC) & $\eta_{0}$, Pa s \\
\hline PS442 & 2 & 141 & 135 & 2.1 & 0.45 \\
PS448 & 2 & 692 & & 2.5 & 65 \\
PS042 & 0 & 153 & \multirow{2}{*}{3.99} & 2.3 & 0.49 \\
T1915 & 4 & & & &
\end{tabular}

a Compositional variables include the functionality, $f$, the initial degree of polymerization, $X_{\mathrm{n}}$, the polydispersity, $\mathrm{PD}$, and the zeroshear viscosity of the material, $\eta_{0}$, as determined by dynamic mechanical experiments.

HULS Petrarch and were devolatilized at $140{ }^{\circ} \mathrm{C}$ for $14 \mathrm{~h}$ in vacuo before use to remove trace low molecular weight derivatives as well as water, which may cause side reactions. ${ }^{19}$ Their properties are shown in Table II. The molecular weight was determined by GPC with a PDMS standard (number-average degree of polymerization, $X_{\mathrm{n}}=130$ ) and by vinyl titration. ${ }^{20}$ The silane was purified in a spinning band distillation column to achieve a purity determined by gas chromatography to be better than $99.95 \%$. The average functionality as determined by titration was $3.99 \pm 0.05$. The catalyst, cis-dichlorobis(diethyl sulfide)platinum, was dissolved in toluene at concentrations on the order of $10^{-5}-10^{-6} \mathrm{~mol}$ of $\mathrm{Pt} / \mathrm{mol}$ of toluene. This solution was mixed with the prepolymer in a molar ratio between $10^{-3}$ and $10^{-4} \mathrm{~mol}$ of $\mathrm{Pt} / \mathrm{mol}$ of vinyl to provide a convenient reaction time $(1-3 \mathrm{~h})$ to the gel point. Prepolymer, cross-linker, and catalyst were then mixed in an atmosphere of nitrogen. Bubbles introduced by the mixing dissolved before rheological study.

This system was chosen since (i) the polymer and cross-linker are readily available in an array of molecular weights and functionalities, (ii) the initial compounds are well characterized and can be obtained with a high degree of purity, (iii) the kinetics of the catalyzed reaction can be controlled easily at room temperature, and (iv) the material shows no evidence of competing phase transitions or vitrification in the experimental frequency window throughout the entire reaction. A disadvantage of PDMS may be its initial broad molecular weight distribution. No information is available on the effect of polydispersity on gels.

Rheological Experiments. Small-strain oscillatory shear measurements were performed by using a Rheometrics RDS7700 dynamic spectrometer with parallel-plate geometry. All samples were reacted isothermally to within $\pm 0.2 \mathrm{~K}$ at room temperature. Strains between 0.02 and 0.5 were applied to provide an accurate torque response (at least $0.1 \%$ of maximum torque). The material was shown to behave linearly at these levels. ${ }^{21}$ Venkataraman and Winter ${ }^{22}$ have shown that strains on the order of 2 and above lead to disruption of the molecular network.

Chambon and Winter ${ }^{1}$ determined the critical gel properties by quenching several different trials at different extents of reaction close to GP. They could not obtain a material precisely at the GP by this method because of the critical nature of the transition. A more direct way of determining the critical gel properties is to characterize the material during the cross-linking reaction without stopping it. However, characterization of transitory materials necessarily reduces the frequency window that may be probed because time-temperature superposition experiments are not possible and because a rapid material change does not allow probing at low frequency. This is no problem for our application, since the critical gel has a very simple rheological behavior that can be easily distinguished from viscoelastic liquid or solid behavior, and thus a wide window of frequency is not required. The limits of power law behavior are interesting and worthy of further study, but they were not the goal of this work. Our samples did not exhibit competing transitions, and a significant frequency window was well accessible. As long as the frequencies were chosen in the terminal zone of the prepolymer, we always observed an intermediate power law state for the gel point.

For studying the transient network polymer, Holly et al..$^{24}$ suggested the Fourier transform mechanical spectroscopy method of probing several frequencies simultaneously. Here, however, 


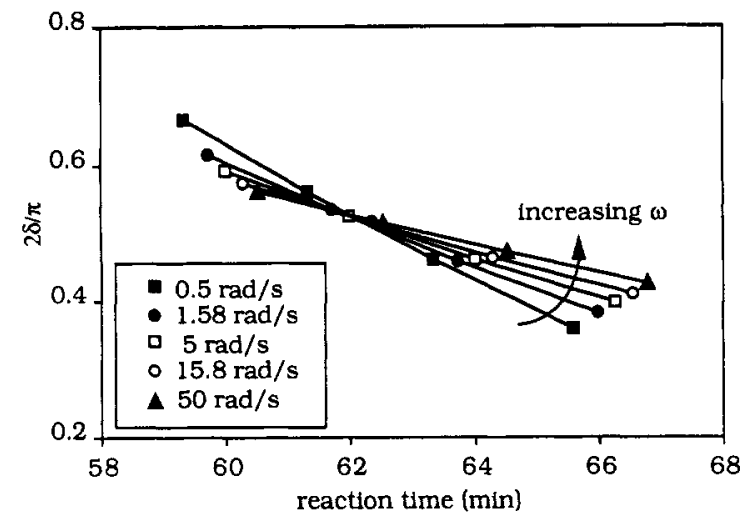

Figure 1. Evolution of the normalized phase angle as a function of reaction time at five frequencies. The gel point occurs close to $62 \mathrm{~min}$.

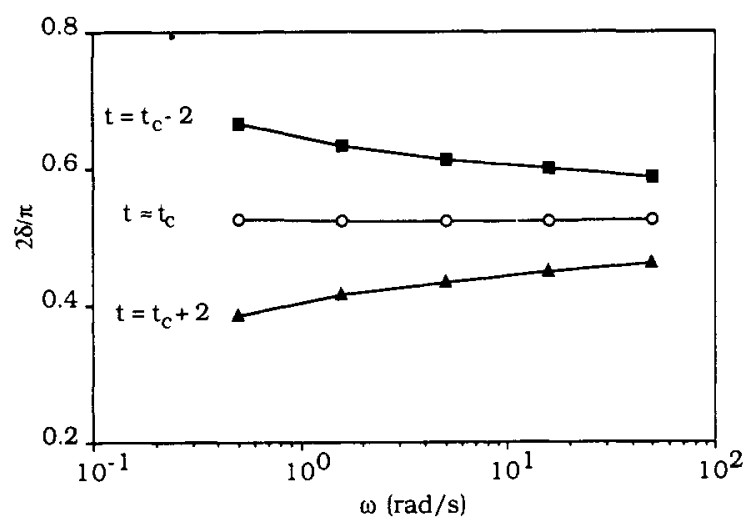

Figure 2. Interpolated values normalized phase angle as a function of frequency at three times. The symbol $\Delta$ represents $t-t_{\mathrm{c}}=+2 \mathrm{~min}, 0$ represents $t-t_{\mathrm{c}}=0 \mathrm{~min}$, and $\mathbf{a}$ represents $t-t_{c}=-2 \mathrm{~min}$.

we chose to probe each frequency individually to minimize the strain on the material and to expand the available frequency window. ${ }^{23}$ A series of frequency sweeps during the cross-linking of the sample gave modulus data at various frequencies and various states in the vicinity of the gel point. Interpolation of these data gave properties at discrete material states, including the gel point, which is easily detected by its self-similarity $(\delta=$ constant over the entire frequency range). Results obtained by the in situ technique compare favorably to results obtained on stopped samples..$^{1-3}$

A typical evolution of the phase angle at five different frequencies as a function of reaction time is shown in Figure 1 for a typical PDMS near the GP. The critical point occurs at $61.9 \mathrm{~min}$. Shown in Figure 2 are interpolated values of the phase angle as a function of frequency at three different times near the GP. Liquid behavior is clearly evident in the phase-angle response at $t-t_{\mathrm{c}}=-2 \mathrm{~min}$, as is the solid behavior at $t-t_{\mathrm{c}}=+2 \mathrm{~min}$. The relaxation exponent is calculated as $n=0.52 \pm 0.01$. The dynamic modulus also demonstrates power law behavior, and the gel strength is calculated as $S=900 \pm 75 \mathrm{~Pa} \mathrm{~s}^{n}$.

The experiments were performed at room temperature, which was an uncontrollable parameter and over the course of this work varied by $7 \mathrm{~K}$. Winter and Chambon ${ }^{2}$ showed that the temperature dependence of the gel strength follows

$$
S(T)=S\left(T_{0}\right)\left(a_{T}^{n} / b_{T}\right)
$$

PDMS properties obey an Arrhenius-type temperature dependence because it is far above the glass transition temperature $\left(-125^{\circ} \mathrm{C}\right)$. The temperature shift factors are

$$
a_{\mathrm{T}}=\exp \left\{\frac{E}{R}\left(\frac{1}{T}-\frac{1}{T_{0}}\right)\right\} ; \quad b_{\mathrm{T}}=\frac{\rho\left(T_{0}\right) T_{0}}{\rho(T) T}
$$

From the shift factors determined by Winter and Chambon, we may estimate that room-temperature fluctuations affect the gel strength by no more than $5 \%$.

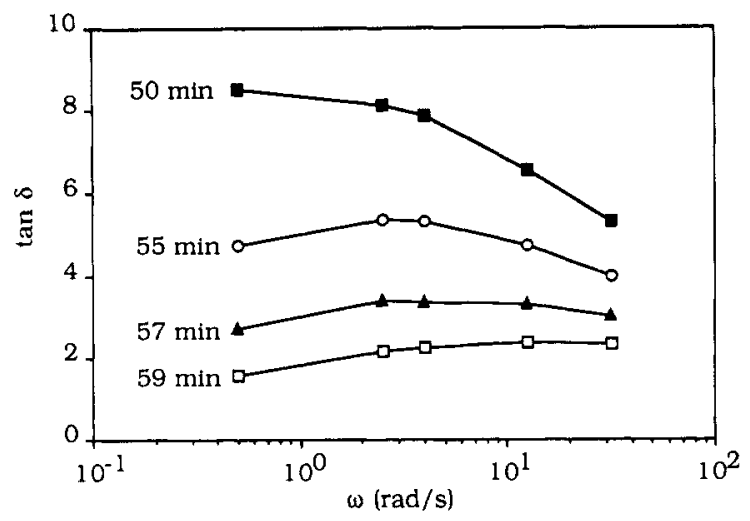

Figure 3. Evolution of the phase angle for an inhomogeneous material through the liquid-solid transition. Different regions of the material reach the gel point at different times.

Perhaps underappreciated in the gelation literature is the importance of temperature homogeneity during reaction. Parts of a nonisothermal sample pass through the gel point at different times, and the material is only locally self-similar. Because the torque integrates the material response over a macroscopic sample volume, small temperature gradients that arise in the air-heated sample chamber can lead to a smearing of the mechanical selfsimilarity. Figure 3 illustrates this behavior with a typical crosslinking polymer. Shown is $\tan \delta$ at several times near the gel point. It is impossible to determine an instant of self-similarity (gel point) from this experiment. However, with careful temperature control, this problem could be minimized. Occasionally, samples with too much inhomogeneity would arise uncontrollably, so experiments with the uncertainty of the relaxation exponent greater than \pm 0.03 in the frequency window of 2 decades were rejected and then repeated until within the tolerance. The material was probed for only a short time near the gel point. Liquid behavior was clearly evident before the experiment, as was solid behavior after experiment completion. With the proper precautions, we found the in situ measurement of $\tan \delta$ to be a precise technique for determining the GP.

\section{Results}

Medium-Chain-Length Prepolymer. Winter et al. ${ }^{13}$ showed that the stoichiometry influences the critical gel properties. For a polyurethane of $X_{n} \sim 17$, the relaxation exponent took on values

$$
\begin{gathered}
1 / 2 \leq n \leq 2 / 3, \quad r<1 \\
n \approx 1 / 2, \quad r>1
\end{gathered}
$$

The relaxation exponent appeared to be sensitive to a deficiency of cross-linker but insensitive to an excess of cross-linker. The maximum in the gel strength was thought to occur near the maximum imbalanced stoichiometry, $r_{\mathrm{u}}$, rather than at balanced stoichiometry, where most final network properties have an extremum. ${ }^{25}$

We have repeated this experiment on a different prepolymer, a medium-chain-length PDMS prepolymer, PS442, $X_{\mathrm{n}} \approx 135$, with a four-functional cross-linker, T1915. The gel strength and relaxation exponent for this system are shown in Figure 4 as a function of stoichiometry. Each pair of $S$ and $n$ represents a different experiment. The relaxation exponent takes on a range of values of $0.4 \leq n \leq 0.7$. The results obtained here compare favorably with earlier studies of Chambon and Winter; however, there appears to be a minimum in the relaxation exponent as a function of stoichiometry when there is an excess of cross-linker, that is, $r>1$. The gel strength varies by about 1 order of magnitude and is also sensitive to a deficiency of cross-linker but relatively insensitive to 

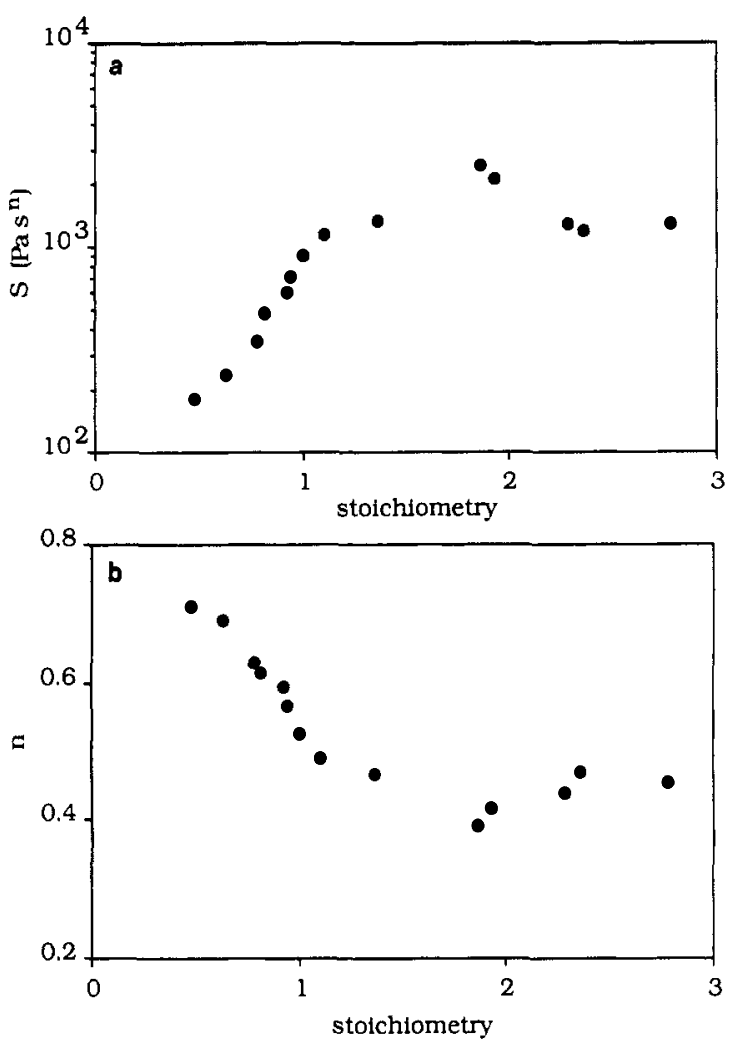

Figure 4. (a) Gel strength and (b) relaxation exponent as a function of stoichiometry $(r=$ moles of cross-linker ends $/$ moles of prepolymer ends) for the medium-chain-length prepolymer, PS442.

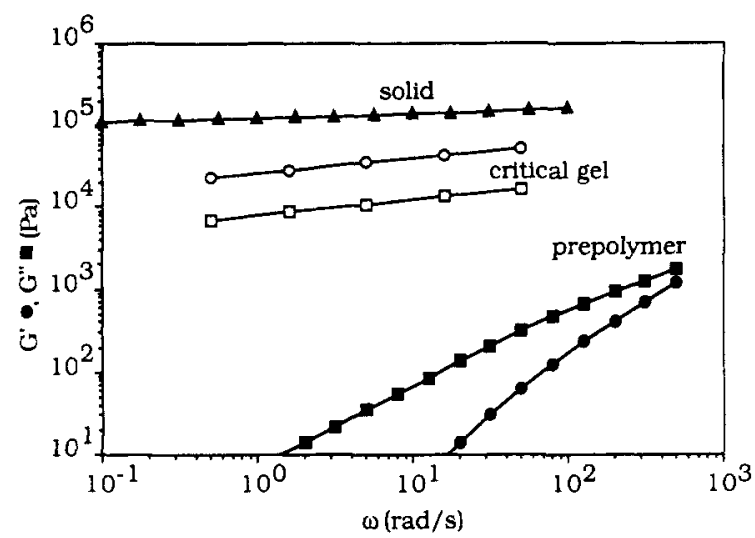

Figure 5. Frequency response of the dynamic mechanical properties, the loss modulus, $G^{\prime \prime}(\square)$, and storage modulus, $G^{\prime}$ (O), for the high molecular weight prepolymer, PS448 (unfilled symbols), and critical gel (filled symbols) at a stoichiometry of $r=1.3$. Also shown is the modulus of the final network, $G^{\prime}(\Delta)$ (the loss modulus is left off the figure for clarity).

a deficiency of polymer. We find, however, a maximum in the gel strength between $r=1$ and $r_{u}$. Surprisingly, the shape of the gel strength mirrors the relaxation exponent, suggesting that the two properties may be coupled.

High-Chain-Length Prepolymer. The role of chain length and entanglements were tested by using a longchain-length prepolymer, PS448, with $X_{\mathrm{n}}=692$. The chain length is approximately 5 times greater than the entanglement molecular weight $\left(M_{c}=24500^{26}\right)$. This polymer also exhibits a power law state during gelation. The linear viscoelastic properties of the CG for this material are shown in Figure 5 as determined by the in situ method. For comparison the linear viscoelastic properties of the prepolymer and final network rubber are also shown. A value of $n=0.19 \pm 0.01$ is found for this sample.
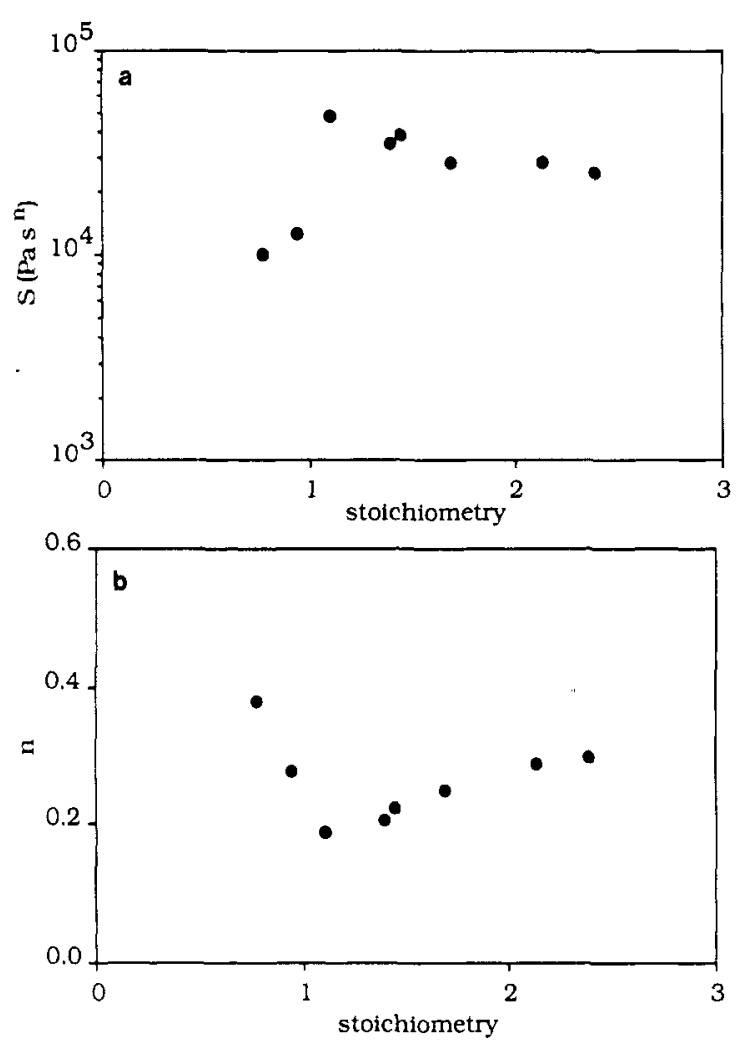

Figure 6. (a) Gel strength and (b) relaxation exponent as a function of stoichiometry for the high molecular weight prepolymer, PS448.

While this polymer displays a power law region at GP, the presence of entanglements was expected to produce different dynamical behavior at high frequencies, i.e., when probing length scales smaller than a characteristic distance between entanglements. This polymer, however, does not exhibit a plateau region, which is evidence of entangled behavior, in the observable frequency window.

The results for PS448 at different stoichiometries are summarized in Figure 6. This polymer also has a minimum in the relaxation exponent function, which occurs near $r$ $=1.1$. The gel strength varies by about 1 order of magnitude and again mirrors the relaxation exponent. The relaxation exponent for the long-chain-length prepolymer is lower, on the order of $0.2-0.4$, than that for the short-chain-length PU or the medium-chain-length PDMS. Thus both chain length and stoichiometry influence critical gel properties.

Dilution of Medium-Chain-Length Polymer. The presence of a diluent during gelation was studied with the medium-chain-length prepolymer, PS442, and an inert PDMS (PS042) of nearly the same initial molecular weight $\left(X_{\mathrm{n}} \sim 153\right)$. A material with the same chemical composition was chosen to avoid polymer-solvent interactions that might lead to phase separation. The study was carried out at constant stoichiometry, $r=1.7$, which is near where the extremum of the stoichiometric dependence of the CG properties occurs for this polymer. The diluent was a Newtonian fluid with a viscosity of $0.49 \mathrm{~Pa}$ s.

Solutions with polymer concentrations as low as the overlap concentration, $\phi^{*}$, were studied. At the overlap concentration, the crossover between dilute and semidilute polymer concentrations occurs, and it is here that the ends of the growing clusters are just able to meet to form an infinite network. At reaction completion there is only one cluster, the infinite cluster, which is swollen by the solvent. Several different compositions were made 


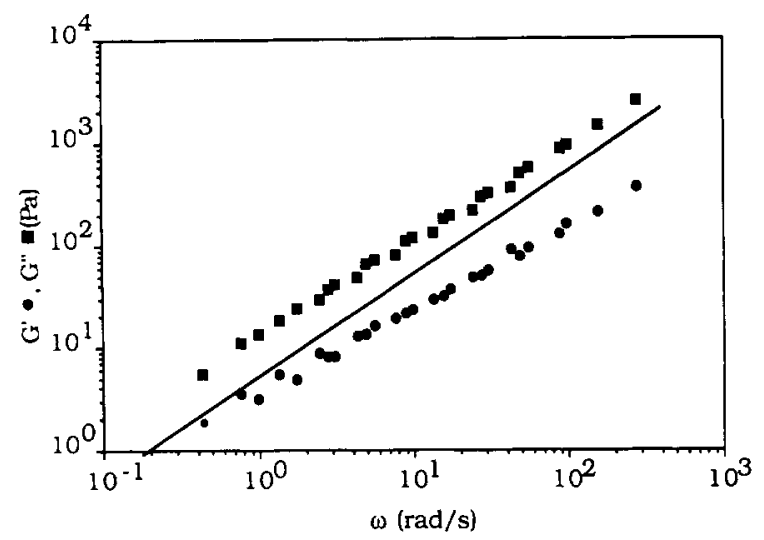

Figure 7. Dynamic mechanical properties for a near critical gel of a PS442/PS042 blend at a concentration of $95.2 \%$ by weight PSO42 with a stoichiometry $r=1.7$. The line represents the loss modulus of the pure diluent (note that the diluent has no elastic component).

to determine the critical composition, which for this polymer and stoichiometry was found to occur at $\approx 5.2 \mathrm{wt}$ $\%$ polymer. Rheological properties of a completely reacted sample near $\phi^{*}$ are shown in Figure 7 and are compared to the dynamic properties of the pure diluent. The rheological properties for this material were measured at three temperatures $\left(+90,+25\right.$, and $\left.-25^{\circ} \mathrm{C}\right)$ and then shifted by time-temperature superposition to obtain a master curve. The rheological properties indicate that this material is slightly beyond the gel point, but it is clear that it passed through a power law state, even at this extremely low polymer concentration. We estimate from the highfrequency response a value of the relaxation exponent as $n=0.92 \pm 0.04$.

The concentration dependence of the critical gel properties for the PS442/PS042 blend is shown in Figure 8. All compositions down to $\phi^{*}$ exhibit a mechanically self-similar state during the evolution of cross-linking. Dilution, however, dramatically affects the critical gel properties. The relaxation exponent takes on all values between the value at the undiluted state and a value close to the upper limit of 1 . In fact, it is experimentally difficult to distinguish between a Newtonian fluid and a critical gel with $n \approx 1$. The relaxation exponent decreases with increasing dilutent concentration above about 25 wt $\%$ diluent. This change of behavior may be indicative of a crossover from concentrated to semidilute polymer concentrations. For this blend, the ratio of viscous/elastic character of the $\mathrm{CG}$ changes with the addition of diluent by 1 order of magnitude from 1 to 10 .

Dilution of High-Chain-Length Polymer. The effect of dilution was also studied on the high-chain-length polymer, PS448, diluted with the same PDMS solvent, PS042. The trend of the CG properties is qualitatively similar to that of the medium-chain-length polymer, as presented in Figure 9. The relaxation exponent, insensitive to low diluent concentrations, begins to increase when the diluent concentration approaches $\sim 50 \%$. This change in character occurs at a higher concentration than for the medium-strand prepolymer, but this is consistent with the speculation of a crossover from concentrated to semidilute conditions, which is molecular weight dependent. We expect that, at lower polymer concentrations, the relaxation exponent would take on high values, similar to the medium-chain-length polymer.

\section{Discussion}

The data presented here challenge the notion of a universal value of the relaxation exponent. Data between
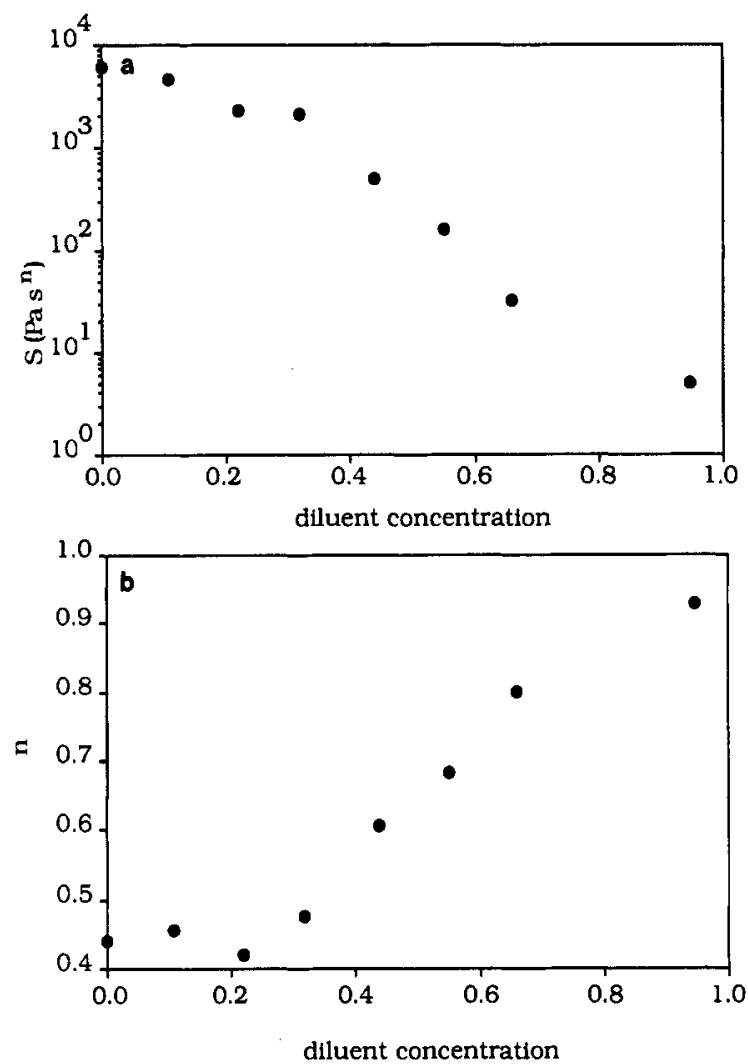

Figure 8. (a) Gel strength and (b) relaxation exponent as a function of concentration of polymer for the PS442/PS042 blend.
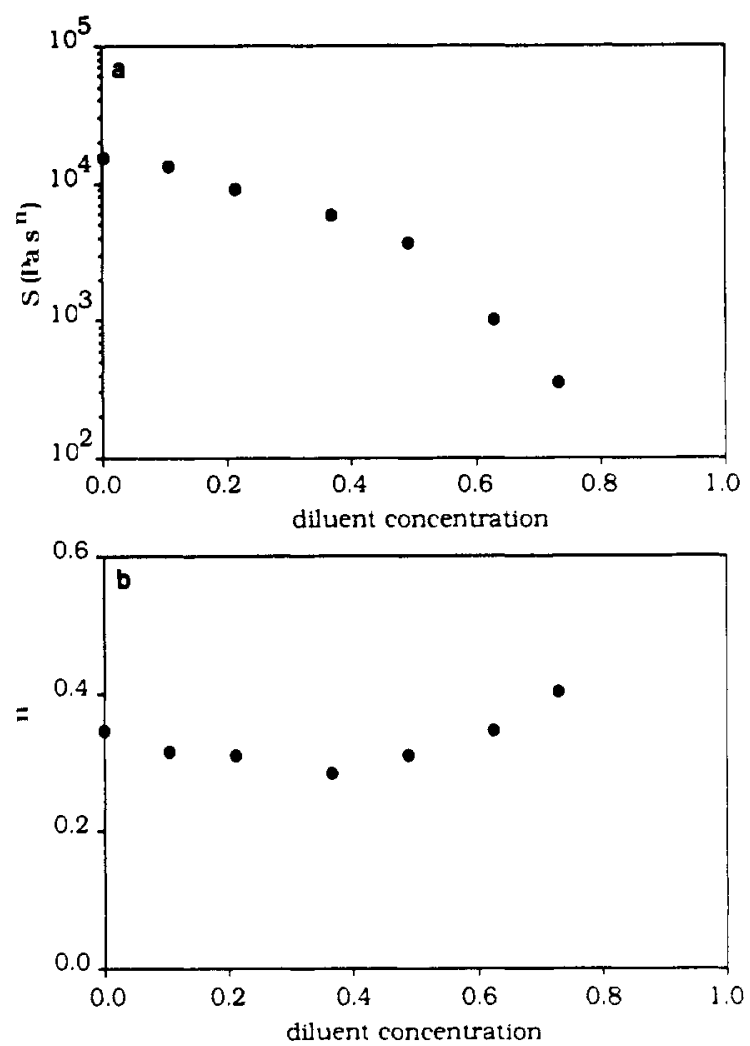

Figure 9. (a) Gel strength and (b) relaxation exponent as a function of concentration of polymer for the PS448/PS042 blend.

0.19 and 0.92 have been reported in this study. This raises doubt as to whether theories of the dynamical behavior described above (i.e., eqs 6,7 , and 10) can predict all of our observations. Tests of the above equations are not yet possible, because the fractal dimension has not yet been measured in the reaction bath. ${ }^{27}$ Our results can be 
described qualitatively, however, if the fractal dimension does not assume a universal value. In this case though, several theories in combination with our data would predict fractal dimensions outside the range $1 \leq d_{\mathrm{f}} \leq 3$. The prediction of Muthukumar, eq 10, does yield values of the fractal dimension in the physical range for all values of $n$. According to this theory, the screening of excluded volume leads to a fractal dimension different from the percolation prediction, and degrees of screening may produce values between those predicted by eqs 8 and 10. Qualitatively, this theory helps to explain several of our observations. CGs with high values of the relaxation exponent have low fractal dimensions and are said to be "open". CGs with lower relaxation exponents have higher fractal dimensions and are "tight". Screening of excluded volume may increase as the molecular weight of the $C G$ increases, compacting these materials. Diluted materials are swollen, and as dilution increases the cluster expands and the fractal dimension decreases.

Winter ${ }^{28}$ showed that the relaxation exponent is related to the exponents that characterize the divergence of the zero-shear viscosity and the equilibrium modulus, $s$ and $z$, respectively, by

$$
n=z /(z+s)
$$

This relation comes solely from experimental considerations. A variable $n$, therefore, suggests that the exponents $s$ and $z$ are also not universal.

Since the relaxation exponent is not universal, it may be wiser to correlate viscoelastic properties to structural parameters other than just the critical exponents. The static structure exponents are difficult to measure, although advances in determining the polydispersity index have been made, ${ }^{29}$ and it appears to be universal. A critical gel property that may be useful for correlation is the number-average molecular weight at the gel point $M_{\mathrm{n}, \mathrm{c}}$, which is continuous through the critical region. $M_{\mathrm{n}, \mathrm{c}}$ normalizes the molecular weight distribution at the gel point:

$$
N_{\mathrm{M}}=\left(M / M_{\mathrm{n}, \mathrm{c}}\right)^{-\tau} \text {, at GP }
$$

$M_{\mathrm{n}, \mathrm{c}}$ many be measured or predicted by theory. ${ }^{30}$ It is a function of all of the precursor compositional variables

$$
M_{\mathrm{n}, \mathrm{c}}=f\left(M_{f}, M_{g}, \mathrm{PD}, f, g, r, \phi\right)
$$

where $f$ and $g$ are functionalities, $M_{f}$ and $M_{g}$ are the molecular weights, PD is the polydispersity, $r$ is the stoichiometry, and $\phi$ is the concentration. $M_{n, c}$ has a maximum as a function of stoichiometry at $r \geq 1$. The extremum of the relaxation exponent may occur at this stoichiometry. The low values of the relaxation exponent found for a vulcanized polyethylene ${ }^{16}$ and the crystallizing polypropylene ${ }^{17}$ can also be qualitatively explained in this manner. These processes lead to clusters with high functionality at GP and thus a high $M_{\mathrm{n}, \mathrm{c}}$.

All random cluster growth models predict a universal value of the fractal dimension, but perhaps gelation is more like colloidal aggregation, ${ }^{31}$ where the cluster structure depends strongly on the rules of cluster growth. Martin et al ${ }^{32}$ suggested that for long-strand prepolymers not all sites on a cross-linker molecule would be equally accessible, especially after one or more of the sites has reacted. This transport-limited phenomena might be described by nonrandom percolation models. There is evidence to suggest that the reaction pathway also influences the critical gel properties, and temperature (sample inhomogeneity) and large-strain ${ }^{22}$ effects have already been demonstrated. Dependence of the critical gel properties on the kinetics

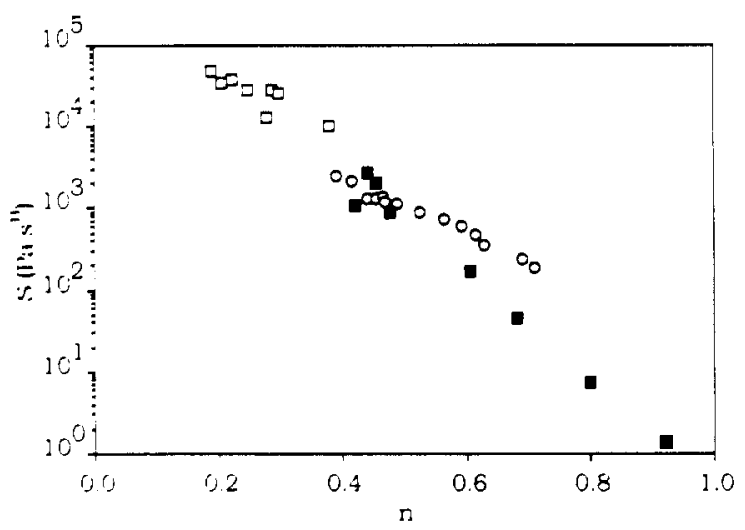

Figure 10. Gel strength as a function of the relaxation exponent for PS442 (O) and PS448 (a) and the PS442/PS042 blend ( $(\square)$. The results of a least-squares fit of the data are shown in Table III.

Table III

Values of the Time Constant and Modulus Determined by Least-Squares Fit of the Data in Figure 10

\begin{tabular}{lccc}
\hline \multicolumn{1}{c}{ system } & $G_{0}, \mathrm{~Pa}$ & $\lambda_{0}, \mathrm{~s}$ & $G_{0} \lambda_{0}, \mathrm{~Pa} \mathrm{~s}$ \\
\hline PS442 & $4.68 \times 10^{4}$ & $4.71 \times 10^{-4}$ & 22 \\
PS448 & $1.91 \times 10^{5}$ & $4.57 \times 10^{-4}$ & 87 \\
PS442/PS042 & $1.15 \times 10^{6}$ & $3.72 \times 10^{-7}$ & 0.43
\end{tabular}

of the reaction would indicate nonrandom effects. Our experiments were designed to minimize the effect of the reaction pathway, but its importance is not yet thoroughly understood.

All of the studies performed here indicate that the gel strength and relaxation exponent are coupled. The data are recast in Figure 10 with the independent variable removed and suggest a simple relation

$$
S=G_{0} \lambda_{0}^{n}
$$

The nature of the unusual dimensions of $S\left(\mathrm{~Pa} \mathrm{~s}^{n}\right)$ becomes clear: $S$ is composed of a short time constant $\lambda_{0}$ and a modulus $G_{0}$. The strength may be replaced in eq 1:

$$
G(t)=G_{0}\left(t / \lambda_{0}\right)^{-n}
$$

A least-squares fit of the strength/relaxation exponent data for a given material determines the time constants. Values for each material can be calculated and are reported in Table III. The results suggest that $G_{0}$ and $\lambda_{0}$ are characteristic of the prepolymer. For the high-chainlength polymer, PS448, $G_{0}$ is $1.9 \times 10^{5} \mathrm{~Pa}$, which is very close to the plateau modulus, $G_{\mathrm{N}^{0}}{ }^{0}$, which is $2.11 \times 10^{5} \mathrm{~Pa}$ for PDMS. ${ }^{18}$ Since the relaxation exponent is limited to $0<n<1$, eq 19 indicates that the gel strength is constrained to

$$
G_{0} \lambda_{0}<S<G_{0}
$$

where the changing $n$ not only affects the magnitude of $S$ but also its dimension. We speculate that the upper limit is given by the plateau modulus and the lower limit by the viscosity of the linear precursor that forms the network strands. The precursor viscosity may be compared to that of a Maxwell liquid with a single relaxation time

$$
\eta_{0}=G \lambda
$$

The constants determined by our experiments yield viscosities that are reasonably close to the actual material viscosities reported in Table II, but further experimentation is required to confirm this relation. 


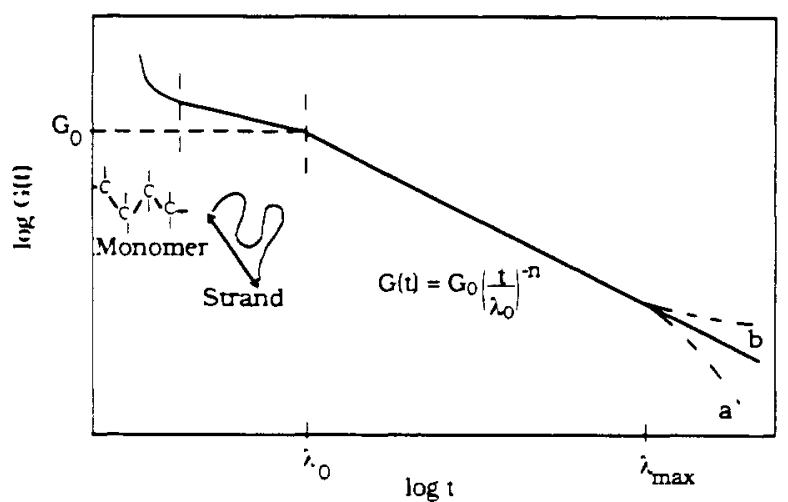

Figure 11. Schematic representation of the relaxation modulus indicating the range of power law for a material near the gel point. The very short time behavior associated with the monomer and strand motions is also depicted. $G_{0}$ and $\lambda_{0}$ indicate the short time limit of power law behavior. The long time limit is given by the finite sample dimensions, although a practical limit for a material is given by the longest relaxation time, $\lambda_{\max }$, which diverges in the vicinity of the gel point. Dashed lines indicate (a) liquid behavior and (b) solid behavior.

There are natural limits to the range of power law behavior as sketched in Figure 11. It is typical for polymeric liquids and solids that the very short time relaxation behavior is dominated by the motion of bonds and, at a slightly longer time scale, by the motion of monomer units and of entire strands. ${ }^{26}$ This behavior differs from the behavior of the critical gel. It clearly marks the short time limit of the power law region. On the other side, no long time limit of the power law behavior is expected, except by the finite sample size. For our experiments, there are practical limits to the long time window since a real sample is either a liquid or solid.

The transition to short-time dynamical behavior was not accessible to us with the polymers studied here. It might be possible to explore this transition with a prepolymer with a larger time constant, $\lambda_{0}$. Dilution studies might also lead to different dynamical behavior at high frequency, where motions associated with the solvent rather than those of the infinite cluster would be observed.

The dependence of the gel strength on molecular weight seems to be different below and above the entanglement molecular weight. Chambon et al. ${ }^{33}$ found that $S$ decreases with $M_{\mathrm{n} 0}$ for small molecular weight materials $\left(M_{\mathrm{n} 0}\right.$ was in the range $500-2000$ for the PU system studied). As the cross-link density decreases with increasing $M_{\mathrm{n} 0}$, the strength decreases (for constant $n$ ). However, for higher molecular weights, $S$ increases with increasing $M_{\mathrm{n} 0}$, which may indicate the presence of trapped entanglements.

The gel strength also has a strong concentration dependence. The data are plotted on a log-log scale to reveal regions of sealing behavior (Figure 12). The behavior at intermediate concentration, perhaps indicative of the semidilute region, is distinguished by

$$
S \sim \Phi^{a}
$$

For comparison, the final equilibrium shear modulus (determined by dynamic mechanical means at a constant frequency of $0.1 \mathrm{rad} / \mathrm{s}$ ) of the medium-weight polymer is also shown. The final modulus scales with a concentration at a power close to 2 . The gel strength, on the other hand, has a higher concentration dependence. For the highweight polymer the exponent $a$ is of the order 3 . For the medium-weight polymer, the exponent $a$ is of the order $4-5$. We are limited to a rather short range of concentration, so rigorous conclusions about scaling are not possible.

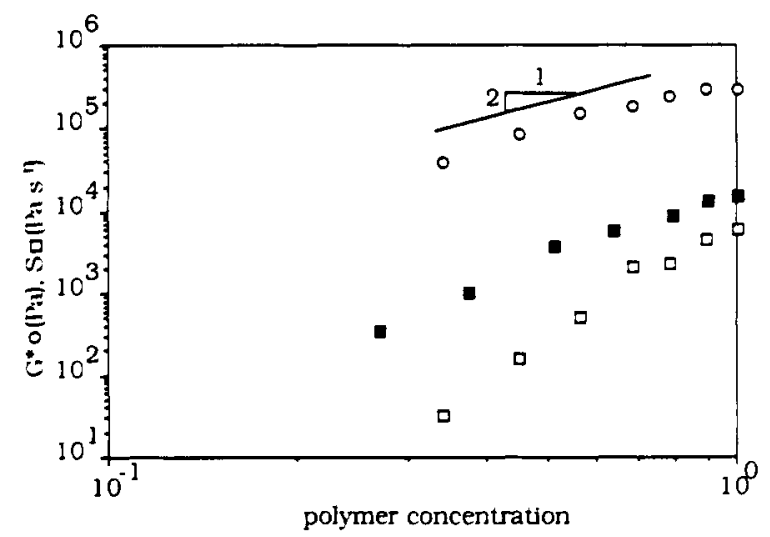

Figure 12. Gel strength for PS442 (ם) and PS448 (a) vs the polymer concentration. Also shown is the dynamic mechanical modulus at $0.1 \mathrm{rad} / \mathrm{s}$ for PS442 (O) after completion of the reaction.

\section{Conclusions}

We have further evidence that, in the absence of competing phase transitions, the material at the gel point exhibits power law rheological behavior. The critical gel rheological properties, however, are not universal. Values of the relaxation exponent reported in this study, $0.19 \leq$ $n<0.92$, have been established over almost the entire possible range, and the gel strength is seen to vary by 5 orders of magnitude. Stoichiometry, molecular weight, and concentration were all shown to have an impact on the critical gel properties. For the polymers studied here, the CG properties are coupled. The coupling relation defines the material constants $G_{0}$ and $\lambda_{0}$.

This study suggests that rheological experiments alone will not help to distinguish between the popular theories of gelation. While the linkage process may be universal, the dynamical properties depend strongly on the material composition and structure. Studies involving concurrent determination of the static exponents, $d_{f}$ and $\tau$, and the dynamic exponent, $n$, are required to distinguish between the various theories.

From a practical point of view, it is advantageous that critical gel properties are not universal but instead depend on molecular structure. It allows us to prepare materials near the gel point with a wide range of properties for applications such as adhesives, absorbants, vibration dampers, sealants, membranes, and others. By proper molecular design, it will be possible to tailor network structures, relaxation character, and the strength of gels to one's requirements.

Acknowledgment. We gratefully acknowledge the National Science Foundation for its support under Grants NSF-MSM86-01595 and NSF-DMR-88-06556. We also thank M. Muthukumar, S. K. Venkataraman, and Y. G. Lin for valuable discussions.

\section{References and Notes}

(1) Chambon, F.; Winter, H. H. Polym. Bull. (Berlin) 1985, 13, 499.

(2) Winter, H. H.; Chambon, F. J. Rheol. 1986, 30 (2), 367.

(3) Chambon, F.; Winter, H. H. J. Rheol. 1987, 31 (8), 683.

(4) Stauffer, D.; Coniglio, A.; Adam, M. Adv. Polym. Sci. 1982, 44, 103.

(5) Muthukumar, M. J. Chem. Phys. 1985, 83 (6), 3161.

(6) Cates, M. E. J. Phys. (Les Ulis, Fr.) 1985, 46, 1059.

(7) Muthukumar, M.; Winter, H. H. Macromolecules 1986, 19, 1284

(8) Durand, D.; Delsanti, M.; Adam, M.; Luck, J. M. Europhys. Lett. 1987, 3 (3), 97.

(9) Martin, J. E.; Adolf, D.; Wilcoxon, J. P. Phys. Rev. Lett. 1988, $61(22), 2620$. 
(10) Hess, W.; Vilgis, T. A.; Winter, H. H. Macromolecules 1988, 21 , 2536.

(11) Muthukumar, M. Macromolecules 1989, 22 (12), 4656.

(12) Treloar, L. R. G. The Physics of Rubber Elasticity, 3rd ed.; Clarendon Press: Oxford, 1975.

(13) Winter, H. H.; Morganelli, P.; Chambon, F. Macromolecules $1988,21,532$.

(14) Rubinstein, M.; Colby, R. H.; Gilmor, J. R. Polym. Prepr. (Am. Chem. Soc., Div. Polym. Sci.) 1989, 30, 81.

(15) Nijenhuis, K.; Winter, H. H. Macromolecules 1989, 22, 411.

(16) Valles, E. M.; Carella, J. M.; Winter, H. H.; Baumgaertel, M. Rheol. Acta, in press.

(17) Lin, Y. G.; Mallin, D. T.; Chien, J.C.W.; Winter, H. H., submitted for publication in Macromolecules.

(18) Valles, E. M.; Macosko, C. W. Macromolecules 1979, 12, 521.

(19) Fisher, A.; Gottlieb, M. Proceedings of Networks 86, Elsinor Denmark, Aug 1986.

(20) Smith, A. L. Analysis of Silicones; John Wiley and Sons: New York, 1962.

(21) Venkataraman, S. K. Ph.D Thesis, University of Massachusetts, Amherst, MA, 1990.
(22) Venkataraman, S. K.; Winter, H. H. Rheol. Acta 1990, 29, 423

(23) Scanlan, J. C. Ph.D Thesis, University of Massachusetts, Amherst, MA, 1990.

(24) Holly, E. E.; Venkataraman, S. K.; Chambon, F.; Winter, H. H. J. Non-Newtonian Fluid Mech. 1988, 27, 17.

(25) Leung, Y. K.; Eichinger, B. E. J. Chem. Phys. 1984, 80, 3887.

(26) Ferry, J. D. Viscoelastic Properties of Polymers; John Wiley and Sons: New York, 1980.

(27) Martin, J. E.; Keefer, K. D. Phys. Rev. 1986, A34 (6), 4988.

(28) Winter, H. H. Prog. Colloid Polym. Sci. 1987, 75, 104.

(29) Schosseler, F.; Benoit, H.; Grubbisic-Gallot, Z.; Strazielle, $\mathrm{Cl}$; Leibler, L. Macromolecules 1989, 27, 400.

(30) Macosko, C. W.; Miller, D. R. Macromolecules 1976, 9, 199.

(31) Meakin, P. Adv. Colloid Interface Sci. 1988, 28, 249.

(32) Martin, J. E., Adolf, D.; Wilcoxon, J. P. Phys. Rev. A 1989, 39, 1325.

(33) Chambon, F.; Petrovic, Z. S.; MacKnight, W. J.; Winter, H. H. Macromolecules 1986, 19, 2146.

Registry No. cis-Dichlorobis(diethyl sulfide)platinum, 1544257-6; tetrakis (dimethylsiloxy)silane, 17082-47-2. 